EISSN: $2706-7947$ ISSN: 2077- 4613

DOI: 10.36632/mejas/2021.11.1.26

Journal homepage: www.curresweb.com

Pages: 323-333

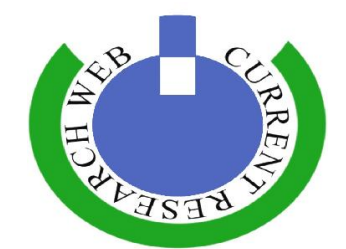

\title{
Incorporated Untraditional Sources of Roughage in Growing New Zealand White, NZW) Rabbit Rations and Its Effects on Nutrient \& Cell Wall Digestibility and Nutritive Values
}

\author{
Roshdy I. El-Kady ${ }^{1}$, Hamed A.A. Omer ${ }^{1}$, Sawsan M. Ahmed ${ }^{1}$, Walid S. El-Nattat ${ }^{2 *}$ Aly \\ A. El-Shahat ${ }^{1}$, Mahmoud Y. El-Ayek ${ }^{3}$ and Ashraf A. A. Morad ${ }^{1}$ \\ ${ }^{\text {I} A n i m a l ~ P r o d u c t i o n ~ D e p a r t m e n t, ~ N a t i o n a l ~ R e s e a r c h ~ C e n t r e, ~} 33$ El-Buhouth Street, P.O: 12622, Dokki, \\ Cairo, Egypt. \\ ${ }^{2}$ Animal Reproduction and Artificial Insemination Department, National Research Centre, 33 El- \\ Buhouth Street, P.O: 12622, Dokki, Cairo, Egypt \\ ${ }^{3}$ Animal Production Department, Faculty of Agriculture, Mansoura University, Mansoura, Egypt.
}

Received: 16 January $2021 \quad$ Accepted: 15 March $2021 \quad$ Published: 25 March 2021

\begin{abstract}
Seventy eight rabbits (4-5wks) divided into 13 groups that contained 6 were used in thirteen digestibility trials to study the availability of using rice straw (RS) or corn stalks (CS) as untraditional roughage sources as a partial or complete substitution or replacement for clover hay . Replacing clover hay $(\mathrm{CH})$ that incorporated in control at $33 \%$ of ration formulation by RS treated with or without Pleurotus ostreatus and CS with or without Trichoderma reesei at levels (0, 11, 22 and 33\%).Untreated RS (UBTRS) or biological treated (BTRS) with Pleurotus ostreatus caused an increasing in their contents of crude protein (CP), nitrogen free extract (NFE) and Ash while CF, OM, NDF, ADF, ADL and hemicellulose decreased in comparable with the raw rice straw. On the other hand, biological treatment of CS (BTCS) with Trichoderma reesei occurred a decreasing in their values of $\mathrm{OM}$, crude fiber (CF), NFE, NDF, ADF, cellulose and hemicellulose, meanwhile, CP, ash and ADL contents of (BTCS) and untreated CS (UBTCS) were increased. All dietary treatments significantly increased OM digestibility. The best value of OM digestibility was recorded by rabbits fed 33\% BTRS. Rabbits fed $22 \%$ and $33 \%$ BTRS or $22 \%$ UBTCS and $11 \%$ BTCS significantly $(\mathrm{P}<0.05)$ increased CP digestibility. Rabbits fed $33 \%$ (UBTRS or BTRS) and significantly increased CF digestibility. The best value of EE digestibility was recorded by rabbits that fed 33\% BTCS. All dietary treatments significantly $(\mathrm{P}<0.05)$ increased NFE digestibility. All dietary treatments significantly $(\mathrm{P}<0.05)$ increased TDN value. Also, Rabbits received $22 \%$ and $33 \%$ BTRC and $22 \%$ UBTCS \& $11 \%$ BTCS significantly $(\mathrm{P}<0.05)$ increased their value of DCP. The highest value of NDF digestibility was recorded by rabbits fed $11 \%$ BTCS, meanwhile the lowest value was observed with 33\% UBTCS $(\mathrm{P}<0.05)$. Rabbits fed 11, 22 and 33\% BTCS occurred significantly $(\mathrm{P}<0.05)$ increasing in hemicellulose digestibility.
\end{abstract}

Keywords: Rice straw, corn stalk, rabbit, Pleurotus ostreatus, Trichoderma reesei

\section{Introduction}

Several crops produced large amounts of stalks, cobs, straws.....etc, can be use as untraditional source of Berseem or Berseem hay. Field crops content largely present digestible nutrients (El- Shahat et al., 2006; Omer et al. 2012, Kholif et al., 2014).

In Egypt, amount of wheat straw, rice straw and corn stalks are completely used for feeding animals (Ministry of Agric and Land Reclamation 2008).

Sadek (2013) reported that agriculture by-products about 35 million tons annually, 23 million tons of which were plant wastes ( 7 million tons of them are used as fodders, 4 million tons were used as organic fertilizers and the rest of wastes which 12 million tons were left without any using).

Corresponding Author: Walid S. El-Nattat, Animal Reproduction and Artificial Insemination Department, National Research Centre, 33 El-Buhouth Street, P.O: 12622, Dokki, Cairo, Egypt.

E-mail: dr.ade176@gmail.com 
Limiting factors of using by-products for feeding animals are low protein content (3-4\%); high crude fiber (30-45\%); Cellulose (30-40\%); hemicellulose (5-35\%) approximately; lignin (10-15\%), approximately, poor palatability and low digestibility (Thander and Aman 1984).

Previous work mentioned that, crop residues contain 30-40\% cellulose, $16-27 \%$ hemicelluloses and 3-13 lignin, meanwhile the wood residues contain 45-56\% cellulose, 10-25\% hemicellulose and 18-30\% lignin, respectively (Chahal 1985).

Straws treated by white-rot fungi may be a good method to increase their nutritive quality (Fazaeli et al. 2002).Also fungi are responsive for lignocellulose degradation are fungi, and the most effective are basidiomycetes (Rabinovich et al. 2004). Enzymes released from fungi break the polysaccharidelignin and this improve degradation of straws, which may make straws more digestible. This would enhance the accessibility of enzymes to potentially digestible biomass resulting in higher degradation of the straw, which may create a more nutritious feed (Tawffek 2011).

Reducing indigestible cell wall and increasing the digestion coefficients of cell wall of straws may be due to role of action of Pleurotus ostreatus (Fazaeli et al. 2002).

Treated rice straw with Pleurotus ostreatus in sheep rations caused significantly increasing in digestibility of DM, OM, CP, CF, NDF, ADF, ADL, cellulose, hemicellulose, and nutritive values includes TDN and DCP (Abd-ElAziz and Ismail 2001).

Also, Deraz and Ismail (2001) noticed that all nutrients digestibility of cotton stalks and nutritive values as TDN and DCP were improved as a result of biological treatments, especially with Coriolus versicolor or Pleurotus ostreatus, followed by Ph. chrysosporium, compared with untreated cotton stalks.

This study was carried to invistigate the effect of incorporation untreated or treated biologically of both rice straw by Pleuratus ostreatus and corn stalks by Trichoderma reesei on nutrient and cell wall digestibility of rabbits.

\section{Materials and Methods}

\subsection{Methods}

The experimental work was carried out at El-Nubaria Experimental and Production Station, ElImam Malik Village, Behira Governorate.

This work designed to study the effect of cultivation Pleurotus ostreatus on RS and Trichoderma reesei on CS and replacing clover hay by untreated and treated RS and CS at different levels $(0,33,66$ and $100 \%)$.

\subsection{Biological treatments}

\subsubsection{Microorganisms:}

Trichoderma reesei and Pleurotus ostreatus were obtained from Agriculture Microbiology Department, National Research Centre, Dokki, Cairo, Egypt.

\subsubsection{Biological treatments of crop residues under study in large scale}

A heap of $160 \mathrm{~kg}$ of the each tested chopped and crushed rice straw or corn stalks were moistened with medium contained, $2.5 \%$ molasses, $2.5 \%$ urea, $1.5 \%$ ammonium sulphate, $1.00 \%$ supper phosphate and $0.5 \%$ magnesium sulphate at solid: liquid (1:2). The treated crop residues were shuffled upside down daily for the prop inoculation period (14 days). At the end of fermentation period, the treated crop residues were collected and exposed to sun- dry until the moisture content reached less than $10 \%$ then packed and stored until used in manufacturing the pelleted feed. About $80 \mathrm{~kg}$ of rice straw or corn stalks were treated with above solution (without Pleuratus ostreatus or Trichoderma reesei).

\subsection{Manufacturing the pelleted feed}

The air dried compost have been transported to the forage manufacture for making the experimental pelleted feed by substituting of clover hay with biologically treated and untreated rice straw or corn stalks at the levels of (33\% and $66 \%$ and $100 \%)$. 


\subsection{Animals and feeds}

Seventy eight weaned New Zealand white rabbits (4-5 weeks) and about $565 \pm 13.57 \mathrm{~g}$ were randomly divided into thirteen experimental groups of 6 rabbits in each. Each group divided into three replicates of two rabbits in each.

All the experimental diets were approximalty iso-nitrogenous and iso-caloric that meets requirements of growing rabbits according to (NRC, 1977) recommendation.

Each one of the thirteen experimental group rabbits received one of the following ration:

$\mathrm{R}_{1}$ : Experimental ration contained 33\% clover hay and considered as control.

$\mathrm{R}_{2}$ : Experimental ration contained $11 \%$ rice straw (RS) without fungi (UBTRS).

$\mathrm{R}_{3}$ : Experimental ration contained $22 \%$ rice straw (RS) without fungi (UBTRS).

$\mathrm{R}_{4}$ : Experimental ration contained 33\% rice straw (RS) without fungi (UBTRS).

$\mathrm{R}_{5}$ : Experimental ration contained $11 \%$ rice straw (RS) treated by Pleurotus ostreatus fungi (BTRS).

$\mathrm{R}_{6}$ : Experimental ration contained 22\% rice straw (RS) treated by Pleurotus ostreatus fungi (BTRS).

$\mathrm{R}_{7}$ : Experimental ration contained 33\% rice straw (RS) treated by Pleurotus ostreatus fungi (BTRS).

$\mathrm{R}_{8}$ : Experimental ration contained $11 \%$ corn stalks (CS) without fungi (UBTCS).

$\mathrm{R}_{9}$ : Experimental ration contained 22\% rice straw (RS) without fungi (UBTCS).

$\mathrm{R}_{10}$ : Experimental ration contained 33\% rice straw (RS) without fungi (UBTCS).

$\mathrm{R}_{11}$ : Experimental ration contained $11 \%$ corn stalks (CS) treated by Trichoderma reesei fungi (BTCS).

$\mathrm{R}_{12}$ : Experimental ration contained $22 \%$ corn stalks (CS) treated by Trichoderma reesei fungi (BTCS).

$\mathrm{R}_{13}$ : Experimental ration contained 33\% corn stalks (CS) treated by Trichoderma reesei fungi (BTCS).

All animals were kept under the same managerial and hygienic conditions and were housed in metal battery cages two rabbits in each. Each replicate involved two rabbits were housed separately in metal cages and provide with feed and water ad-libitum at $27-28^{\circ} \mathrm{C}$ ambient temperature with natural light and ventilation.

\subsection{Digestibility trials}

Thirteen digestibility trials were carried out using all rabbits $(6$ rabbits in three replicates of two rabbits) in each treatments were used from each treatment to determine the nutrient and cell wall digestibility and calculated nutritive value of (total digestible nutrient, TDN and digestible crude protein, DCP) of the experimental diets. All calculations of digestibility trials was carried out according to the classical method that described by Abou-Raya (1967).Feed and water were offered ad libitum.

Feed intake and excreted feces were daily recorded for 5 consecutive days before the end of experiment. Composite samples of dried feces (10\%) were dried for $48 \mathrm{hrs}(60)$, ground and stored for chemical analysis.

Chemical analysis of feeds and dried feces was determined according to AOAC (2005).

\subsection{Analytical procedures}

Samples of ingredients, diets and dried feces were analyzed according to AOAC (2005) methods for dry matter (DM), ether extract (EE). Crude protein (CP), Crude fiber (CF) and ash. The nitrogen free extract (NFE) and organic matter (OM) content were calculated. Fiber fractions includes (NDF, ADF and ADL) were determined according to Goering and Van Soest (1970) and Van Soest et al. (1991). Hemicellulose and cellulose contents were calculated by difference using the following equations:

Hemicellulose $=$ NDF-ADF Cellulose $=$ ADF-ADL .

\subsection{Statistical analysis}

Data were statistically analyzed as one way analysis of variance using the general linear model procedure of SPSS (2008).

Meanwhile, Duncan's Multiple Range Test was used to examine the significance between means, Duncan (1955). 


\section{Results}

\subsection{Chemical analysis of different ingredients}

Untreated or BTRS caused an increasing in their level of crude protein, NFE and ash in comparison with the raw rice straw. The portion of improving values were recorded as the follows; crude protein increased by $178.75 \%$ and $224.5 \%$; NFE $6.30 \%$ and $24.53 \%$ and ash $22.73 \%$ and $44.45 \%$. Meanwhile, CF content depressed by $31.32 \%$ and $56.75 \%$, but OM content reduced by $2.81 \%$ and $5.51 \%$. BTCS cause an decrease in OM, CF, NFE, NDF, ADF, cellulose and hemicellulose while CP, ash and ADL were increased (Table 1).

Table 1: Chemical analysis of different roughages used in ration formulations

\begin{tabular}{|c|c|c|c|c|c|c|c|}
\hline \multirow[t]{2}{*}{ Item } & \multirow{2}{*}{$\begin{array}{c}\text { Clover } \\
\text { hay, } \\
\text { CH) }\end{array}$} & \multicolumn{3}{|c|}{ Rice straw (RS) } & \multicolumn{3}{|c|}{ Corn stalks (CS) } \\
\hline & & Raw & UBTRS & BTRS & Raw & UBTCS & BTCS \\
\hline Moisture (\%) & 13.88 & 6.70 & 7.50 & 8.00 & 7.00 & 8.50 & 8.20 \\
\hline \multicolumn{8}{|c|}{ Chemical analysis on DM basis (\%) } \\
\hline OM & 89.10 & 89.00 & 86.50 & 84.10 & 88.50 & 87.01 & 84.69 \\
\hline $\mathbf{C P}$ & 17.03 & 5.27 & 14.69 & 17.10 & 5.61 & 13.99 & 16.68 \\
\hline CF & 14.07 & 45.66 & 31.36 & 19.75 & 37.78 & 32.96 & 24.13 \\
\hline $\mathbf{E E}$ & 2.50 & 1.10 & 1.15 & 1.21 & 1.11 & 1.32 & 1.56 \\
\hline NFE & 55.50 & 36.97 & 39.30 & 46.04 & 44.00 & 38.74 & 42.32 \\
\hline Ash & 10.90 & 11.00 & 13.50 & 15.90 & 11.50 & 12.99 & 15.31 \\
\hline \multicolumn{8}{|c|}{ Cell wall constituents $(\%)$} \\
\hline NDF & 33.84 & 68.75 & 65.75 & 61.99 & 72.50 & 65.85 & 60.00 \\
\hline ADF & 18.17 & 47.50 & 45.15 & 43.75 & 56.00 & 47.75 & 45.25 \\
\hline ADL & 12.89 & 14.85 & 13.56 & 10.99 & 15.56 & 16.95 & 18.75 \\
\hline Hemicellulose & 15.67 & 21.25 & 20.60 & 18.24 & 16.50 & 18.10 & 14.75 \\
\hline Cellulose & 5.28 & 32.65 & 31.59 & 32.76 & 40.44 & 30.80 & 26.50 \\
\hline
\end{tabular}

UBTRS: Unbiological treated rice straw by Pleurotus ostreatus fungi.

BTRS: Biological treated rice straw by Pleurotus ostreatus fungi.

UBTCS: Unbiological treated corn stalks by Trichoderma reesei fungi.

BTCS: Biological treated corn stalks by Trichoderma reesei fungi.

Hemicellulose $=$ NDF- ADF. Cellulose $=$ ADF- ADL .

\subsection{Chemical analysis of the experimental diets}

$\mathrm{R}_{11}$ contains higher persent of $\mathrm{CP}$ and hemicellulose ,however the highest valuse of cellulose was noticed with $\mathrm{R}_{10}$ meanwhile $\mathrm{R}_{\mathbf{1}}$ recorded higher level of $\mathrm{CF}, \mathrm{ADF}$ and $\mathrm{ADL}$. Concerning about DE, $\mathrm{R}_{9}$ had the best value (Table 2).

\subsection{Nutrient digestibility of the experimental groups}

Data of Table (3) mentioned that DM digestibility was insignificantly increased when rabbits fed ration containing $11 \%$ BTCS $\left(\mathrm{R}_{11}\right)$, meanwhile rabbits fed 11 or $33 \%$ UBTRS $\left(\mathrm{R}_{2}\right.$ and $\left.\mathrm{R}_{4}\right)$ and $33 \%$ UBTCS $\left(\mathrm{R}_{10}\right)$ caused significant $(\mathrm{P}<0.05)$ decreasing in DM digestibility comparing to the control. On the other hand, rabbits fed $\left(R_{3}, R_{5}\right.$ to $R_{9}, R_{12}$ and $\left.R_{13}\right)$ occurring insignificantly decreasing in their $D M$ digestibility in comparison with the control. All dietary treatments significantly increased OM digestibility compared to control, the best value of OM digestibility (77.51\%) was recorded by rabbits fed $33 \%$ BTRS $\left(\mathrm{R}_{7}\right)$. Rabbits fed rations containing $22 \%$ and $33 \%$ BTRS, $22 \%$ UBTCS or $11 \%$ BTCS $\left(\mathrm{R}_{6}, \mathrm{R}_{7}, \mathrm{R}_{9}\right.$ and $\left.\mathrm{R}_{11}\right)$ significantly $(\mathrm{P}<0.05)$ increased $\mathrm{CP}$ digestibility compared to control. Rabbits fed $33 \%$ UBTRS $\left(\mathrm{R}_{4}\right)$ or $33 \%$ BTRS $\left(\mathrm{R}_{7}\right)$ significantly increased CF digestibility compared to control. The best value of EE digestibility was recorded by rabbits that fed $33 \%$ BTCS $\left(\mathrm{R}_{13}\right)$ in comparison with the control, the corresponding value of $E E$ digestibility was (96.06 vs. $85.65 \%$ ) for $R_{13}$ and $R_{1}$, respectively. All dietary treatments significantly $(\mathrm{P}<0.05)$ increased NFE digestibility in comparison with the control. The best value of NFE digestibility $(85.11 \%)$ was recorded by rabbits that fed $22 \%$ BTRS $\left(\mathrm{R}_{6}\right)$ that fed $11 \%$ BTCS $\left(\mathrm{R}_{11}\right)$. 
Middle East J. Appl. Sci., 11(1): 323-333, 2021

EISSN: 2706-7947 ISSN: 2077-4613

Table 2: Chemical analysis of the experimental rations

\begin{tabular}{|c|c|c|c|c|c|c|c|c|c|c|c|c|c|}
\hline \multirow[t]{4}{*}{ Ingredients } & \multirow{3}{*}{$\begin{array}{c}\text { Clover } \\
\text { hay } \\
\text { (CH) }\end{array}$} & \multicolumn{6}{|c|}{ Rice straw (RS) } & \multicolumn{6}{|c|}{ Corn stalks (CS) } \\
\hline & & \multicolumn{3}{|c|}{$\begin{array}{l}\text { Unbiological treated rice } \\
\text { straw by Pleurotus ostreatus } \\
\text { fungi (UBTRS) }\end{array}$} & \multicolumn{3}{|c|}{$\begin{array}{l}\text { Biological treated rice straw } \\
\text { by Pleurotus ostreatus fungi } \\
\text { (BTRS) }\end{array}$} & \multicolumn{3}{|c|}{$\begin{array}{l}\text { Unbiological treated corn } \\
\text { stalks by Trichoderma reesei } \\
\text { fungi (UBTCS) }\end{array}$} & \multicolumn{3}{|c|}{$\begin{array}{l}\text { Biological treated corn stalks } \\
\text { by Trichoderma reesei fungi } \\
\text { (BTCS) }\end{array}$} \\
\hline & & $11 \%$ & $22 \%$ & $33 \%$ & $11 \%$ & $22 \%$ & $33 \%$ & $11 \%$ & $22 \%$ & $33 \%$ & $11 \%$ & $22 \%$ & $33 \%$ \\
\hline & $\mathrm{R}_{1}$ & $\mathrm{R}_{2}$ & $\mathrm{R}_{3}$ & $\mathrm{R}_{4}$ & $\mathrm{R}_{5}$ & $\mathrm{R}_{6}$ & $\mathrm{R}_{7}$ & $\mathrm{R}_{8}$ & $\mathrm{R}_{9}$ & $\mathrm{R}_{10}$ & $\mathrm{R}_{11}$ & $\mathrm{R}_{12}$ & $\mathrm{R}_{13}$ \\
\hline Moisture (\%) & 13.88 & 10.05 & 9.78 & 90.83 & 7.38 & 9.17 & 7.52 & 8.79 & 8.90 & 8.89 & 8.46 & 8.57 & 9.03 \\
\hline \multicolumn{14}{|c|}{ Chemical analysis DM basis (\%) } \\
\hline Organic matter (OM) & 74.25 & 88.11 & 87.45 & 86.03 & 89.24 & 86.08 & 83.76 & 90.14 & 89.86 & 88.45 & 88.98 & 88.64 & 87.93 \\
\hline Crude protein (CP) & 17.03 & 17.05 & 17.02 & 17.03 & 17.21 & 17.21 & 17.76 & 16.99 & 17.02 & 17.09 & 18.11 & 16.95 & 16.57 \\
\hline Crude fiber (CF) & 14.07 & 13.39 & 12.59 & 12.16 & 12.20 & 10.76 & 8.58 & 13.37 & 12.56 & 12.56 & 11.38 & 10.36 & 10.07 \\
\hline Ether extract (EE) & 2.50 & 2.36 & 2.23 & 2.29 & 2.35 & 2.15 & 2.30 & 2.56 & 2.39 & 2.28 & 2.57 & 2.32 & 2.50 \\
\hline Nitrogen free extract (NFE) & 40.65 & 55.31 & 55.61 & 54.55 & 57.48 & 55.96 & 55.12 & 57.22 & 57.89 & 56.52 & 56.92 & 59.01 & 58.79 \\
\hline Ash & 25.75 & 11.89 & 12.55 & 13.97 & 10.76 & 13.92 & 16.24 & 9.86 & 10.14 & 11.55 & 11.02 & 11.36 & 12.07 \\
\hline \multicolumn{14}{|c|}{ Calculated $*$} \\
\hline Digestible energy (DE) & 2513 & 2496 & 2491 & 2453 & 2493 & 2445 & 2444 & 2503 & 2595 & 2412 & 2518 & 2501 & 2425 \\
\hline Calcium (Ca) \% & 1.08 & 1.03 & 0.98 & 1.02 & 0.90 & 0.92 & 0.97 & 1.04 & 1.01 & 1.03 & 0.97 & 0.92 & 0.96 \\
\hline Tryptophane \% & 0.80 & 0.82 & 0.79 & 0.80 & 0.85 & 0.80 & 0.82 & 0.80 & 0.79 & 0.80 & 0.82 & 0.79 & 0.83 \\
\hline Lysine \% & 0.89 & 0.83 & 0.77 & 0.72 & 0.86 & 0.40 & 0.71 & 0.85 & 0.80 & 0.74 & 0.85 & 0.71 & 0.63 \\
\hline Methionine + Cestine \% & 0.55 & 0.55 & 0.56 & 0.57 & 0.55 & 0.53 & 0.57 & 0.57 & 0.57 & 0.58 & 0.56 & 0.46 & 0.54 \\
\hline Sodium \% & 0.20 & 0.19 & 0.18 & 0.17 & 0.19 & 0.79 & 0.18 & 0.19 & 0.18 & 0.17 & 0.17 & 0.18 & 0.17 \\
\hline \multicolumn{14}{|c|}{ Cell wall constituents (\%) } \\
\hline Neutral detergent fiber (NDF) & 33.84 & 30.32 & 28.49 & 25.29 & 24.02 & 28.60 & 26.28 & 30.46 & 32.12 & 28.49 & 37.97 & 35.24 & 31.68 \\
\hline Acid detergent fiber (ADF) & 18.17 & 14.42 & 15.83 & 14.18 & 13.95 & 13.32 & 13.49 & 14.74 & 17.72 & 14.73 & 17.54 & 15.38 & 16.90 \\
\hline Acid detergent lignin (ADL) & 12.89 & 10.83 & 5.40 & 3.63 & 11.59 & 6.12 & 2.81 & 3.84 & 7.22 & 2.99 & 6.52 & 8.56 & 5.88 \\
\hline Hemicellulose ${ }^{* *}$ & 15.67 & 15.90 & 13.11 & 11.11 & 10.07 & 15.28 & 12.79 & 15.72 & 14.40 & 13.76 & 20.43 & 19.86 & 14.78 \\
\hline Cellulose ${ }^{* * *}$ & 5.28 & 3.59 & 10.43 & 10.50 & 2.36 & 7.20 & 10.68 & 10.90 & 10.50 & 11.74 & 11.02 & 6.82 & 11.02 \\
\hline
\end{tabular}

*Calculated according to NRC 1977.

$* *$ Hemicellulose $=$ NDF - ADF

*** Cellulose = ADF - ADL

$\mathrm{DE}=\mathrm{kcal} / \mathrm{kg}$ feed 
Table 3: Effect of incorporating different roughage sources on nutrient \& cell wall constituents digestibility and nutritive values of the experimental groups

\begin{tabular}{|c|c|c|c|c|c|c|c|c|c|c|c|c|c|c|}
\hline \multirow{5}{*}{ Item } & \multirow{4}{*}{$\begin{array}{c}\begin{array}{c}\text { Clover } \\
\text { hay } \\
\text { (CH) }\end{array} \\
\text { Control }\end{array}$} & \multicolumn{6}{|c|}{ Rice straw (RS) } & \multicolumn{6}{|c|}{ Corn stalks (CS) } & \multirow{5}{*}{ SEM } \\
\hline & & \multicolumn{3}{|c|}{$\begin{array}{l}\text { Unbiological treated rice straw } \\
\text { by Pleuratus ostreotus fungi } \\
\text { (UBTRS) }\end{array}$} & \multicolumn{3}{|c|}{$\begin{array}{l}\text { Biological treated rice straw } \\
\text { by Pleurotus ostreatus fungi } \\
\text { (BTRS) }\end{array}$} & \multicolumn{3}{|c|}{$\begin{array}{l}\text { Unbiological treated corn } \\
\text { stalks by Trichoderma reesei } \\
\text { fungi (UBTCS) }\end{array}$} & \multicolumn{3}{|c|}{$\begin{array}{l}\text { Biological treated corn stalks } \\
\text { by Trichoderma reesei fungi } \\
\text { (BTCS) }\end{array}$} & \\
\hline & & 11 & 22 & 33 & 11 & 22 & 33 & 11 & 22 & 33 & 11 & 22 & 33 & \\
\hline & & $\%$ & $\%$ & $\%$ & $\%$ & $\%$ & $\%$ & $\%$ & $\%$ & $\%$ & $\%$ & $\%$ & $\%$ & \\
\hline & $\mathrm{R}_{1}$ & $\mathrm{R}_{2}$ & $\mathrm{R}_{3}$ & $\mathrm{R}_{4}$ & $\mathrm{R}_{5}$ & $\mathrm{R}_{6}$ & $\mathrm{R}_{7}$ & $\mathrm{R}_{8}$ & $\mathrm{R}_{9}$ & $\mathrm{R}_{10}$ & $\mathrm{R}_{11}$ & $\mathrm{R}_{12}$ & $\mathrm{R}_{13}$ & \\
\hline \multicolumn{15}{|c|}{ Nutrient digestibility (\%) } \\
\hline Dry matter (DM) & $83.99^{\mathrm{a}}$ & $76.51^{\mathrm{b}}$ & $78.41^{\mathrm{ab}}$ & $78.11^{\mathrm{b}}$ & $78.53^{\mathrm{ab}}$ & $80.54^{\mathrm{ab}}$ & $81.21^{\mathrm{ab}}$ & $80.73^{\mathrm{ab}}$ & $80.18^{\mathrm{ab}}$ & $76.80^{\mathrm{b}}$ & $84.03^{\mathrm{a}}$ & $79.30^{\mathrm{ab}}$ & $80.94^{\mathrm{ab}}$ & 0.54 \\
\hline Organic matter $(\mathrm{OM})$ & $65.11^{\mathrm{g}}$ & $67.11^{\mathrm{f}}$ & $72.27^{\text {bcde }}$ & $73.91^{\mathrm{b}}$ & $72.61^{\mathrm{bcd}}$ & $76.85^{\mathrm{a}}$ & $77.51^{\mathrm{a}}$ & $70.93^{\mathrm{de}}$ & $71.78^{\text {cde }}$ & $70.57^{\mathrm{e}}$ & $76.23^{\mathrm{a}}$ & $72.99^{\mathrm{bc}}$ & $72.79^{\mathrm{bcd}}$ & 0.57 \\
\hline Crude protein (CP) & $64.52^{\mathrm{bcd}}$ & $63.92^{\text {cd }}$ & $69.50^{\mathrm{abc}}$ & $68.13^{\mathrm{abcd}}$ & $69.56^{\mathrm{abc}}$ & $72.49^{\mathrm{a}}$ & $72.23^{\mathrm{a}}$ & $68.12^{\mathrm{abcd}}$ & $72.88^{\mathrm{a}}$ & $68.73^{\mathrm{abc}}$ & $75.06^{\mathrm{a}}$ & $61.14^{\mathrm{d}}$ & $71.81^{\mathrm{ab}}$ & 0.81 \\
\hline Crude fiber (CF) & $32.15^{\mathrm{bc}}$ & $21.38^{\mathrm{de}}$ & $34.80^{\mathrm{b}}$ & $44.46^{\mathrm{a}}$ & $25.49^{\mathrm{cd}}$ & $38.59^{\mathrm{ab}}$ & $44.67^{\mathrm{a}}$ & $23.40^{\text {de }}$ & $21.59^{\mathrm{de}}$ & $37.54^{\mathrm{ab}}$ & $35.82^{\mathrm{b}}$ & $27.05^{\mathrm{cd}}$ & $16.05^{\mathrm{e}}$ & 1.54 \\
\hline Ether extract (EE) & $85.65^{\mathrm{b}}$ & $85.71^{\mathrm{b}}$ & $88.25^{\mathrm{ab}}$ & $89.44^{\mathrm{ab}}$ & $86.65^{\mathrm{ab}}$ & $88.26^{\mathrm{ab}}$ & $90.53^{\mathrm{ab}}$ & $89.70^{\mathrm{ab}}$ & $83.99^{\mathrm{b}}$ & $91.32^{\mathrm{ab}}$ & $93.52^{\mathrm{ab}}$ & $92.40^{\mathrm{ab}}$ & $96.06^{\mathrm{a}}$ & 0.88 \\
\hline Nitrogen free extract (NFE) & $75.51^{\mathrm{e}}$ & $78.37^{\mathrm{d}}$ & $80.96^{\mathrm{c}}$ & $81.64^{\mathrm{bc}}$ & $82.99^{\mathrm{abc}}$ & $85.11^{\mathrm{a}}$ & $83.73^{\mathrm{ab}}$ & $82.04^{\text {bc }}$ & $81.69^{\mathrm{bc}}$ & $77.64^{\mathrm{de}}$ & $84.00^{\mathrm{ab}}$ & $81.75^{\mathrm{bc}}$ & $81.47^{\mathrm{bc}}$ & 0.46 \\
\hline \multicolumn{15}{|c|}{ Nutritive values $(\%)$} \\
\hline Total digestible nutrients & $51.02^{\mathrm{f}}$ & $61.66^{\mathrm{e}}$ & $65.77^{\mathrm{cd}}$ & $66.42^{\mathrm{bcd}}$ & $67.36^{\text {bc }}$ & $68.53^{\mathrm{b}}$ & $67.50^{\mathrm{bc}}$ & $66.82^{\mathrm{bcd}}$ & $66.93^{\text {bcd }}$ & $65.03^{\mathrm{d}}$ & $70.89^{\mathrm{a}}$ & $66.23^{\text {cd }}$ & $66.81^{\text {bed }}$ & 0.76 \\
\hline (TDN) & $10.99^{\mathrm{cd}}$ & $10.90^{\text {cd }}$ & $11.83^{\mathrm{bc}}$ & $11.60^{\mathrm{bc}}$ & $11.97^{\mathrm{bc}}$ & $12.48^{\mathrm{ab}}$ & $12.83^{\mathrm{ab}}$ & $11.58^{\mathrm{bc}}$ & $12.41^{\mathrm{ab}}$ & $11.75^{\mathrm{bc}}$ & $13.59^{\mathrm{a}}$ & $10.36^{\mathrm{d}}$ & $11.90^{\mathrm{bc}}$ & 0.16 \\
\hline Digestible crude protein (DCP) & & & & & & & & & & & & & & \\
\hline \multicolumn{15}{|c|}{ Cell wall constituents digestibility (\%) } \\
\hline Neutral detergent fiber (NDF) & $60.99^{\mathrm{ab}}$ & $42.71^{\mathrm{de}}$ & $45.98^{\mathrm{d}}$ & $47.27^{d}$ & $43.14^{\mathrm{de}}$ & $55.87^{\mathrm{bc}}$ & $54.22^{\mathrm{c}}$ & $48.39^{\mathrm{d}}$ & $47.17^{\mathrm{d}}$ & $38.55^{\mathrm{e}}$ & $66.03^{\mathrm{a}}$ & $61.63^{\mathrm{ab}}$ & $56.72^{\mathrm{bc}}$ & 1.38 \\
\hline Acid detergent fiber (ADF) & $72.87^{\mathrm{a}}$ & $54.83^{\mathrm{cd}}$ & $49.30^{\text {de }}$ & $55.94^{\mathrm{bcd}}$ & $54.84^{\mathrm{cd}}$ & $57.62^{\mathrm{bcd}}$ & $63.66^{\mathrm{bc}}$ & $41.96^{\mathrm{e}}$ & $51.34^{\mathrm{d}}$ & $42.15^{\mathrm{e}}$ & $64.37^{\mathrm{b}}$ & $52.34^{\mathrm{d}}$ & $53.90^{\mathrm{d}}$ & 1.49 \\
\hline Hemicellulose & $47.22^{\mathrm{bcd}}$ & $31.68^{\mathrm{de}}$ & $42.57^{\text {cde }}$ & $36.20^{\text {de }}$ & $26.92^{\mathrm{e}}$ & $54.35^{\mathrm{abc}}$ & $44.26^{\mathrm{bcd}}$ & $54.42^{\mathrm{abc}}$ & $42.04^{\text {cde }}$ & $34.69^{\mathrm{de}}$ & $67.45^{\mathrm{a}}$ & $68.78^{\mathrm{a}}$ & $59.95^{\mathrm{ab}}$ & 2.37 \\
\hline Cellulose & $69.45^{\mathrm{abcd}}$ & $20.02^{\mathrm{g}}$ & $64.96^{\text {abcde }}$ & $82.41^{\mathrm{a}}$ & $23.25^{\mathrm{g}}$ & $74.12^{\mathrm{ab}}$ & $82.41^{\mathrm{a}}$ & $53.37^{\mathrm{def}}$ & $49.84^{\text {ef }}$ & $56.11^{\text {cdef }}$ & $71.23^{\mathrm{abc}}$ & $40.12^{\mathrm{f}}$ & $59.51^{\text {bcde }}$ & 3.38 \\
\hline
\end{tabular}

a, b, c, d, e, f and g: Means in the same row having different superscripts differ significantly $(\mathrm{P}<0.05)$.

SEM: Standard error of the mean. 


\subsection{Nutritive values of the experimental groups}

All dietary treatments significantly $(\mathrm{P}<0.05)$ increased TDN value comparing to the control. The best value of TDN $(70.89 \%)$ was recorded by rabbits fed $11 \%$ BTCS $\left(\mathrm{R}_{11}\right)$ meanwhile the lowest value $(51.02 \%)$ was recorded by those rabbits that fed on the control $\left(\mathrm{R}_{1}\right)$. On the other hand, the other treatments recorded the intermediate values of TDN that ranged from 61.66 to $68.53 \%$ (Table 3 ).

Also, Rabbits received $22 \%$ and $33 \%$ BTRS $\left(\mathrm{R}_{6}\right.$ and $\left.\mathrm{R}_{7}\right) ; 22 \%$ UBTCS $\left(\mathrm{R}_{9}\right)$ and $11 \%$ BTCS $\left(\mathrm{R}_{11}\right)$ significantly $(\mathrm{P}<0.05)$ increased their value of DCP in comparison with that fed the control $(\mathrm{R} 1)$

The corresponding values of DCP were $12.48,12.83,12.41$ and $13.59 \%$ for $\mathrm{R}_{6}, \mathrm{R}_{7}, \mathrm{R}_{9}$ and $\mathrm{R}_{11}$, respectively. Meanwhile, DCP value of control $\left(\mathrm{R}_{1}\right)$ was $10.99 \%$. The best value of DCP $(13.59 \%)$ was observed when rabbits received $11 \%$ BTCS $\left(\mathrm{R}_{11}\right)$.

\subsection{Cell wall constituents digestibility of the experimental groups}

Data illustrated in Table (3) showed that the highest value of NDF digestibility (66.63\%) was recorded by rabbits fed $11 \%$ BTCS $\left(\mathrm{R}_{11}\right)$, meanwhile the lowest value $(38.55 \%)$ was observed when rabbit fed 33\% UBTCS $\left(\mathrm{R}_{10}\right)$. Furthermore, all dietary treatments significantly $(\mathrm{P}<0.05)$ decreased ADF digestibility among the 12 treatments compared to the control $(72.87 \%)$.

Rabbits fed ration containing $11 \%$ BTRS $\left(\mathrm{R}_{5}\right)$ caused significantly $(\mathrm{P}<0.05)$ decreasing in hemicellulose digestibility (26.92\%) in comparison with the control (47.22\%). Rabbits fed rations containing 11 and $22 \%$ BTCS $\left(\mathrm{R}_{11}\right.$ and $\left.\mathrm{R}_{12}\right)$ occurred significantly $(\mathrm{P}<0.05)$ increasing in their value of hemicellulose digestibility (67.45 and $68.78 \%$ ) for $\mathrm{R}_{11}$ and $\mathrm{R}_{12}$, respectively. However, it was noticed that, rabbits fed ration containing 22 and 33\% BTRS (R6 and R7) or that fed 11\% UBTCS containing ration $\left(\mathrm{R}_{8}\right)$ recorded insignificantly $(\mathrm{P}>0.05)$ increasing in their values of hemicellulose digestibility compared to control $\left(\mathrm{R}_{1}\right)$. The corresponding value of were $\left(54.35,44.26\right.$ and $54.42 \mathrm{vs} .47 .22 \%$ for $\mathrm{R}_{6}$, $\mathrm{R}_{7}, \mathrm{R}_{8}$ and $\mathrm{R}_{1}$ ), respectively. Rabbits fed $33 \%$ UBTRS $\left(\mathrm{R}_{4}\right)$ and $33 \%$ BTRS $\left(\mathrm{R}_{7}\right)$ recorded the highest values $\left(82.41\right.$ and $82.41 \%$ ) of cellulose digestibility, followed by rabbits fed $22 \%$ BTRS $\left(\mathrm{R}_{6}\right)$ and $11 \%$ BTCS $\left(\mathrm{R}_{11}\right)$ that recorded $74.12 \%$ and $71.23 \%$. On the other hand rabbits that fed ration containing $11 \%$ UBTRS $\left(\mathrm{R}_{2}\right)$ or $11 \%$ BTRS $\left(\mathrm{R}_{5}\right)$ recorded the lowest value of cellulose digestibility (20.02 and $23.25 \%$ for $\mathrm{R}_{2}$ and $\mathrm{R}_{5}$, respectively).

\section{Discussion}

Values of chemical analysis of different roughage (Table 1) were in agreement with El-Shahat et al. (2006) who noted that biologically treated corn stalks with Trichoderma viride cause an improvement in both CP and NFE percentages and decreasing in CF content. Also, Durand and Chereau (1988) showed that, Trichoderma viride is characterized by high protein content wide spectrum amino acids composition, low nucleic acids contents, no toxicity, no antibiotic production and its ability to consume different substrates. However, the increase in protein content in biologically treated corn stalks may be due to the release of water soluble sugar from polysaccharides might have led to faster growth of fungus which in turn resulted in higher $\mathrm{CP}$ content, or may be related to the addition of basal mineral media containing nitrogen salts as has been suggested by (Grajek 1988 and Garcia et al. 1993). On the other hand, the decreasing in crude fiber content of biologically treated corn stalks in comparison with untreated may be due to the utilization of $\mathrm{CF}$ by the fungi for their growth since fungi among the microorganisms which have capability in decomposing different agricultural by- products as had been suggested by (Kim et al. 1985). El-Tahan et al. (2003) and El-Tahan and Mohamedi (2005) observed that cultivating mushroom, Agaricus basporius on wheat straw increased its contents of CP and ash, while, DM, OM and CF contents decreased by 231.8, 139.4, 1.2, 20.8 and 63.5\% compared with untreated one, respectively. However, Shakweer (2003) noticed that when rice straw and sugarcane bagasse were treated with either yeast, $S$. cerevisiae plus bacterium, Cellulomonas cellulans, yeast plus fungus, Ph. chrysosporium or with the three organisms together, an increase in CP content and a decrease in CF content.

Meanwhile, data of Table (1) cleared that biological treatment of rice straw caused an decreasing in their values of NDF, ADF, ADL and hemicellulose contents. These results in harmony with those recorded by Bassuny et al. (2005) and Al-Barakel et al. (2007). Furthermore, Fazaeli et al. (2004) found that fungal treatments (five species of Pleurotus fungi, coded $P-21, P-30, P-41, P-60$ and P-90) significantly increased $\mathrm{CP}$ and decreased NDF and ADF contents of wheat straw. However, culture, $P-$ 21 significantly showed lower ability than others to degrade the NDF and ADF content. Meanwhile, 
NFE content of corn stalks was decreased as a result of treatment without or with Trichoderma reesei by control (Table 1). This reduction in NFE may be as a result of using it by microorganism for their growth and reproduction. And these results in agreement with those found by Martin (1977) who recorded that many bacterial genera are capable its utilizing steroids as a sole carbon and energy source, there by degrading steroids completely to $\mathrm{CO}_{2}$ and $\mathrm{H}_{2} \mathrm{O}$. Also, our results in agreement with those found by Abd-Allah (2007) and Abd El-Wahed (2007) who noticed an increasing in CP and ash contents; a decreasing in OM, CF, NDF, ADF and ADL contents when corn cobs and stalks, banana wastes and rice \& wheat straws were treated with $T$. viride, or $S$. cerevisiae or both or corn stover that biologically treated with T. harizianum, or S. cerevisiae or both. Meanwhile, Ashour et al. (1991) examined the applicability of chemical, biological and/ or a combination of both on rice straw, they indicated that, the biological treatment with Humicola fuscotra induced higher increase in protein content $(17.54 \%)$ and decreased the cellulose content by about $(40.29 \%)$, while ash content was almost consistent $(18.98 \%)$ compared with the untreated rice straw $(6.48,45.38$ and $18.87 \%$, respectively). The biological treatment of rice straw pretreated with $\mathrm{Ca}(\mathrm{OH})_{2}$ caused a high increase in protein and cellulose contents (16.31 and $68.18 \%$, respectively) but ash content was decreased (11.21\%).

Our results (Table 3) are closed with Abd El-Hakim et al. (2006) who noted that partial and completely replacement of clover hay by rice straw biologically treated by fungi in growing rabbits diets recorded higher values of all nutrients digestion coefficient. Also, Abd El-Aziz and Ismail (2001) noted that when sheep fed ration contained rice straw treated with Pleurotus ostreatus, the digestibility of DM, OM, CP, CF were significantly increased by 20.9, 20.3, 96.4, 33.0\% compared to control. However Deraz and Ismail (2001) observed that all nutrients digestibility of cotton stalks were improved as a result of biological treatments, especially with Pleurotous ostreatus, compared with untreated cotton stalks. Furthermore, Fazaeli et al. (2004) showed that when bulls fed on wheat straw treated with pleurotus fungi significantly increased digestibility of DM and OM, compared with those fed untreated wheat straw spent rice straw. Fayed et al. (2012) noted a significantly higher $(\mathrm{P}<0.05)$ digestion coefficients of DM, OM and NFE when rams fed potato vines treated with fungi, bacteria (Thermonospora funea) or (Cellulomonace cellulose) in comparison with those fed the control ration. Also, present results in agreement with those obtained by Abd-El- Aziz and Ismail (2001) who reported that when sheep received rations contained rice straw treated with Pleurotus ostreatus, the digestibility of NDF, ADF, cellulose, hemicellulose were significantly increased by 20.9, 36.3, 21.0, 47.5\% comparing to the control. Our results seemed to be near from that obtained by Abd-Allah (2007) who observed that treatment with $T$. reesei and $S$. carevisiae had no effect on digestibility of DM, OM and $\mathrm{CP}$ of corn stalks or rice straw.

The effect of incorporation different sources of roughage (rice straw or corn stalks) that untreated or treated biologically with Pleurotus ostreatus fungi for rice straw or by Trichoderma reesei for corn stalks on nutrient \& cell wall constituents digestibility and nutritive values (Table 3 ) were in agreement with many studies that carried out by Hammouda (1996); Kholif (2001); El-Sayed et al. (2001 and 2002); Abd El-Gawad et al. (2002); Abd El-Malik et al. (2003); El-Ashry et al. (2003); Shakwear (2003); El-Manlawi et al. (2005); Kholif et al. (2005) and Abd El-Hakim et al. (2006) who mentioned that the digestion coefficient tended clearly to be higher when rabbits fed biologically treated rice straw at different levels than that fed untreated rice straw, crop residues or banana wastes. In contrast our results disagreement with those found by Owens and Bergen (1983) and Kholif et al. (2014) who reported that the decreasing occurred in CP digestibility when lactating goats fed rations contained spent rice straw (SRS) of Pleurotus ostreatus basidiomycete at 0.25 (SRS25) or 0.45 (SRS45) (w/w, DM basis) as instead of Egyptian Berseem clover (BC, Trifolium alexandrinum) may be because Berseem clover (BC) contains higher levels of soluble CP compared to SRS, and/or because some of the nitrogen in SRS originates from mycelium and the mushroom fruit body. Mycelium nitrogen may be partly complexes with chitin that would not be easily digested (Fazaeli and Talebian Masoodi 2006). Also, our results near from the results obtained by Singh et al. (1990); Abd-Allah (2007); Fayed et al. (2012) and Kholif et al. (2014) who reported that cell wall digestibility tended clearly to be improved with biologically treatment compared to that untreated substrate wastes.

Our results of biologically treatments on nutritive values were in agreement with those obtained by Abd-El-Aziz and Ismail (2001) who reported that when sheep offered ration contained rice straw treated with Pleurotus ostreatus, the nutritive values as TDN and DCP were significantly increased by 9.0, 17.6 and $16.4 \%$ in comparison with the control. Meanwhile, Deraz and Ismail (2001) noted nutritive 
values as TDN and DCP of cotton stalks were improved as a result of biological treatments, especially with Pleuratous ostreatus, compared with untreated cotton stalks. Also, Abd El-Hakim et al. (2006) noted that partial and completely replacement of clover hay by rice straw biologically treated by fungi in growing rabbits diets recorded higher values of nutritive values as TDN and DCP than that recorded by control. Furthermore, Fayed et al. (2012) showed that rams that fed rations contained potato vines treated with fungi had higher $(\mathrm{p}<0.05) \mathrm{TDN}$ and DCP values in comparison with that fed the control ration.

Results concerning with nutritive value of man effect of roughage source disagreement with those obtained by Abd-Allah (2007) who observed that biologically treatments with $T$. reesei and $S$. carevisiae increased DCP value, but TDN was not affected for corn stalks, meanwhile, treated rice straw resulted an decreased in TDN and did not affect DCP compared to the untreated crop residues.

Also, Hammouda (1996); Kholif (2001); El-Sayed et al. , 2001 and 2002); Abd El-Gawad et al. (2002); Abd El-Malik et al. (2003); El-Ashry et al. (2003); Shakwear (2003); El-Manlawi et al. (2005); Kholif et al. (2005) and Abd El-Hakim et al. (2006) mentioned that the nutritive values tended clearly to be improved when rabbits and other livestock fed biologically treated rice straw at different levels than that fed untreated rice straw, crop residues or banana wastes.

\section{Conclusion:}

Rice straw treated by Pleurotus ostreatus and corn stalks by Trichoderma reesei were safety and improved their chemical analysis, nutrient digestibility and nutritive values.

\section{References}

Abd-Allah, S.A.E., 2007. Biological Treatment of Some By products In Ruminants Feeding. M. Sc. Thesis, Faculty of Agriculture, Al-Azhar University, Egypt.

Abd EI-Wahed, A.R.R., 2007. Chemical and Biological Treatments of Some Agriculture Wastes For Ruminant Nutrition. Ph. D Thesis, Fac Agric., Cairo Univ., Egypt, .

Abd El-Aziz, A.A. and H. Ismail, 2001. Evaluation of rice straw treated with urea solution or fungi (Pleurotus osteratus) for sheep. J. Agric. Sci., Mansoura Univ., 26 (11): 6693-6704.

Abd El-Gawad, A.M., G.M. Khalafalla, I.M.E. Shakweer and W.H. Abd El-Malik, 2002. Effect of mechanical, chemical and biological treatments of rice straw on its digestibility nutritive value and some rumen and blood parameters Recent Technologies in Agriculture Proceeding of the $2^{\text {nd }}$ Congress Faculty of Agriculture Cairo University. 28-30.

Abd El-Hakim A.S., I.M.E. Shakweer and A.A. Azoz, 2006. Effect of using different levels of biologically treated rice straw in growing rabbits diets on growth performance, nutrients digestibility and some physiologies parameters. Egypt. J. of Rabbit science, 16 (2): 191-209.

Abd El-Malik W.H., A.M. Abd El-Gawad, I.M.E. Shakweer, and G.M. Khalafalla, 2003. Improving banana wastes using chemical and biological treatments. Egypt. J. Nutrition and Feeds, 6(special Issue): 1039-1053.

Abou-Raya A.K., 1967. Animal and Poultry Nutrition. $1^{\text {st }}$ Ed. Pub. Dar El-Maarif, Cairo (Arabic text book).

Al-Barakel F., K. Jawasreh and B. Al-Hamed, 2007. The nutritive value of fungal treated industrial and agricultural by-products and its effect on Awassi sheep performance. Egypt. J. Nutrition and feeds, 10 (Special Issue):585-597.

AOAC , 2005. Official Methods of Analysis, $18^{\text {th }}$ ed. Association of Official Analytical Chemists, Washington, DC, USA.

Ashour S.A., A.I. Nagaib, M.A. Zahran and W.M. Eita, 1991. Efficiency of chemical and microbiological treatments in improving the nutritional value of some plant residues, as feed for ruminants. Egyptian J. Microbiology, 26 (3): 307-312.

Bassuny S.M., A.A. Abdel-Aziz, H.J. Abd El-Fatah and M.Y.S. Abdel-Aziz, 2005. Fibrous crop byproduct as feed. 4-Effect of biological treatment for rice straw and bean straw on chemical composition, digestibility and some ruminal and blood constituents of sheep. Egypt. J. Nutrition and Feeds, 8(Special Issue): 541-554. 
Chahal D.S., 1985. Solid state fermentation with Trichoderma reesi for cellulose production. Appl. Environ. Microbial., 49-205.

Deraz T.A.A. and H. Ismail, 2001. Cotton stalks treated with white-rot fungi for feeding sheep. Egypt. J. Nutrition and Feeds, 4 (Special Issue): 423-434.

Duncan DB, 1955. Multiple Range and Multiple (F-test). Biometrics, 11: 1-42.

Durand A. and D., Chereau, 1988. A new pilot reactor for solid-state fermentation: Application to the protein enrichment of sugar beet pulp. Biotechnology and Bioengineering 31: 476-486.

El-Ashry, M.A., A.M. Kholif, M. Fadel, H.A. El-Alamy, H.M. El-Sayed and S.M. Kholif, 2003. Effect of biological treatments on chemical composition, In vitro and In vivo nutrients digestibilities of poor quality roughages. Egyptian Journal of Nutrition and Feeds, 6(2): 113-126.

EL-Manylawi M.A., M.R.M. Ibrahim, F.G. Ahmed, and W.A. Salama, 2005. Performance of growing rabbits fed diets containing geranium and spearmint by-products. Egyptian J of Rabbit Sci. 15 (1): $13-26$.

El-Sayed, H.M., M.A. El-Ashry, H.M. Metwally, M. Fadel and M.M. Khorshed, 2001. Effect of chemical and biological treatments of some crop-residues on their nutritive value: 1- Effect of chemical treatments on chemical composition and in vitro disappearance. Egyptian J. Nutr. and Feeds, 4 (1): 387.

El-Sayed H.M., M.A. El-Ashry, H.M. Metwally, M. Fadel and M.M. Khorshed, 2002. Effect of chemical and biological treatments of some crop-residues on their nutritive value: 3- Digestion coefficient, rumen and blood serum parameters of goats Egypt. J. Nutr. and Feeds 5 (1): 55-69.

El-Shahat A.A., A.H. Hessen, H.A.A. Omer, M.A. Khalafalla, A.A. Morad and M. Fadel, 2006. Effect of feeding biologically treated corn stalks on growth performance and carcass characteristics of growing rabbits. J. Agric. Sci. Mansoura Univ., 31 (10): 6173-6185.

El-Tahan, A.A.H. and Th.F. Mohamdi, 2005. Utilization of mushroom by-products for feeding ruminants. 3- Using mushroom by-products (Agaricus basporius) as a silage for feeding buffaloes. Egypt. J. Nutrition and Feeds, 8(Special Issue):35-47.

El-Tahan A.A.H., G.A. Abd El-Rahman, M.A. Sarhan, and F.F. Abou Ammo, 2003. Utilization of mushroom by-products for feeding ruminants. 2- Utilization of mushroom by-products for feeding sheep. Egypt. J. Nutrition and Feeds, 6(Special Issue):879-890

Fayed, Amal, A., M.H.M. Yacout, A.A. Mahrous, A.A. Hassan and H. Zedan,Afaf, 2012. Improving of nutritive value of potato by biological treatments and its effect on small ruminants production performance. Egypt. J. Nutr. and Feeds, 15 (2): 261-271.

Fazaeli H., and A.R. Talebian Masoodi, 2006. Spent wheat straw compost of Agaricus bisporus mushroom as ruminant feed. Asian-Aust J. Anim. Sci. 19:845-851.

Fazaeli H., Z.A. Jelan, A. Azizi, J.B. Liang, H. Mahmodzadeh and A. Osman, 2002. Effects of fungal treatment on the nutritive value of wheat straw. Malaysian J. Anim. Sci. 7:61-71.

Fazaeli H., H. Mahmodzadeh, A. Aziz, Z.A. Jelan, J.B. Liang, Y. Rouzbehan and A. Osman, 2004. Nutritive value of wheat straw treated with Pleurotus fungi. Asian-Australian J. Anim. Sci., 17 (2): 1681-1688.

Garcia, G., J.F. Galvez and J.C. de Blas, 1993. Effect of substitution of growth performance and on energy and nitrogen efficiency. J. of Animal Science, 71: 1823-1830.

Goering H.K. and P.J. Van Soest, 1970. Forge fiber analysis (apparatus, reagents, procedure and some applications). Agriculture. Hand book 379, USDA, Washington, and DC., USA.

Grajek W., 1988. Production of protein by thermophlic fungi from sugar beet pulp in solid-state fermentation. Bio-Tech. Bio-eng., 32: 255-260.

Hammouda, H.I.A., 1996. Biochemical studies on some agricultural residues. Ph. D. Thesis faculty of Agriculture Ain Shams University Cairo, Egypt.

Israilides, C.J., D. Icanomou, K. Kandylis and P. Nikoyris, 1994. Ferment ability of sugar beet and its acceptability in mice. Bioresource Technology, 47 (2): 97-101.

Kholif A.E., H.M. Khattab, A.A. El-Shewy, A.Z.M. Salem, A.M. Kholif, M.M. El-Sayed, H.M. Gado, M.D. Mariezcurrena, 2014. Nutrient Digestibility, Ruminal Fermentation Activities, Serum Parameters and Milk Production and Composition of Lactating Goats Fed Diets Containing Rice Straw Treated with Pleurotus ostreatus. Asian-Australasian Journal of Animal Sciences (AJAS), 27(3): 357-364. 
Kholif, A.M., M.A. El-Ashry,; H.A. El-Alamy,; H.M. El-Sayed,; M. Fadel, and S.M. Kholif, 2005. Biological treatments of banana wastes for feeding lactating goat. Egypt. J. Nutrition and Feeds, 8(2):149-162.

Kholif S.M.M., 2001. Effect of biological treatments of low quality roughages on milk yield and composition of. Ph. D. Thesis, faculty of Agriculture Ain Shams University Cairo, Egypt.

Kim, J.H., M. Hosobuchi, T. Kishimoto, T. Seki, T. Yoshida, H. Taguchi and D.D.Y. Ryu, 1985. Cellulase production by solid state culture system biotechnology and bioengineering, 27: 14541450.

Martin, D.R., 1977. Effect of buffers upon fiber digestion On Regulation of Acid-Base Balance Symposium. Tucson, Arizona.

Ministry of Agric. and Lank Reclamation, 2008. Part 1. Published by Agric. Res. Center, Egypt. Ministry of Agriculture.

NRC, 1977. National Research Council. Nutrient requirements of rabbits, National Academy of Science, Washington, D.C.

Omer H.A.A., F.A.F. Ali and Sawsan M. Gad, 2012. Replacement of clover hay by biologically treated corn stalks in growing sheep rations. Journal of Agricultural Science, 4 (2): 257-268. Published by Canadian Center of Science and Education.

Owens, F.N., and W.G. Bergen 1983. Nitrogen metabolism of ruminant animals: historical perspective, current understanding and future implications. J. Anim. Sci. 57:498-518.

Rabinovich, M.L., A.V. Bolobova, and Vasil'chenko, 2004. Fungal decomposition of natural aromatic structures and xenobiotics: a review. Appl. Biochem. Microbiol. 40:1-17.

Sadek, Enath, E., 2013. Economic and environmental impacts of using agricultural waste for producing non-traditional feeds. Proceeding of the 14th Scientific Conference of Animal Nutrition, 26-29 November, 2013. Hurghada, Egypt. Egyptian J. Nutrition and Feeds, 16 (2): 139-147.

Shakweer I.M.E., 2003. Effect of biological treatments of rice straw and sugarcane bagasse on their digestibility, nutritive value, ruminal activity and some blood parameters in rams. Egypt. J. Nutrition and Feeds, 6(Special Issue):925-940.

Singh, K., Rai S.N., Rakatan, and Y.W. Han, 1990. Biochemical profiles of solid state fermented wheat straw with Coprinus fimetarius. Indian Journal of Dairy Science, 60:984-990.

SPSS, 2008. Statistical package for Social Sciences, Statistics for Windows, Version 17.0. Released 2008. Chicago, U.S.A.: SPSS Inc.

Tawffek, J.A., 2011. Comparison study between using two kinds of Pleurotus ostreatus mushroom for improving barley straw. J. Food Industries Nutr. Sci. 1:71-74.

Thander, O. and P. Aman, 1984. Straw and other fibrous by products as feed. Chapter 4. Anatomical and Chemical Characteristics. In: Sundstol, F. and E, OW en E. 9 eds). Elsevier Science. Publishers, Amsterdam.

Van Soest, P.J., J.B. Robertson and B.A. Lewis, 1991. Methods for dietary fiber, neutral detergent fiber and non starch polysaccharides in relation to animal performance. J. Dairy Sci., 74: 3583-3597. 